\title{
Percolation Model of Composites: Fraction Clusters and Internal Boundaries
}

\author{
Ale xander Herega ${ }^{1, *}$, Valery Vyrovoy², Alexander Pysare nko ${ }^{1}$ \\ ${ }^{1}$ Department of Phy sics and Mathematical Simulation Laboratory, Odessa State Academy of Civil En gin eering and Architecture, Odessa, \\ 65029, Ukraine \\ ${ }^{2}$ Department of Building Structure Productions, Odessa State Academy of Civil Engineering and Architecture, Odessa, 65029, Ukraine
}

\begin{abstract}
The role of internal boundaries as an integral part of the structure of composite materials is discussed. Computer model of the percolation structure of the composites is proposed. The model used algorithms that based on the Monte-Carlo method is build. The two- and three dimensional composites model is studied and parameters of percolation clusters formed in the model are calculated. The oscillate interactions model of a composite structure components is offered. Analytical exp ressions for an estimation of the process period are received.
\end{abstract}

Keywords Structure, Properties, Fractal Model, Percolation Cluster, Internal Boundaries

\section{Introduction}

Understanding the relationship between structure and properties of matter, understanding of the structure as a model description, which sets the order in the distribution of characteristics values and the law of their evolution[1, 2], can, depending on the task, to focus attention on certain features of the material and neutralize the other. The models considered in this paper, describe the properties of resulting in the first place, the structure of the intermediate asymptotic[3]: in this case come to the fore a cluster component of the material and its internal boundaries (IB) attribute components of the structure of the mesoscopic scale[3-6].

An internal boundary of the material is a fractal surface [4], separating different substances or different phases of one substance, or passing on material defects [7]. (Note that sometimes the internal boundaries are considered in the absence of heterogeneity or qualitative diffe rences.Consider, as an example, the Earth's equator as the boundary between the upper and lower hemispheres of homogeneous sphere, and if allowed zero-dimensional boundaries, then the North Pole, and the centre of gravity, etc. [8]).

The inclusion of IB in the structural parameters of the material has several aspects. First, the IB are the inevitable result disproportionate and macroscopic structural units; second, the material is a complex system and, therefore, must have internal boundaries $[9,10]$.

* Corresponding author:

aherega@gmail.com (Alexander Herega)

Published online at http://journal.sapub.org/cmaterials

Copyright (C) 2012 Scientific \& Academic Publishing. All Rights Reserved
Research connected domains such as interior boundaries, using the methods of percolation theory $[3,4]$ is a relevant for nearly half a century. In such problems, both physical body cluster systems and their influence on the object as a whole are studied. Interest in the percolation clusters (PC) of the particles and the internal boundaries of the material can be easily explained. PC substantially alter the processes of conduction, lead to the anomalous diffusion, determine the mechanical strength and corrosion resistance, impact on longevity, and other physical, chemical and mechanical properties. In addition, near the structural phase transition due to the large size of the clusters, their geometry does not actually depend on the substance, and has universal properties $[3,4,6]$.

In spite of the great influence of the IB on properties of materials, was not studied the process of simultaneous forming of two cluster systems - clusters of particles and clusters of IB. The other two models presented in the article, describe some aspects of the force interaction of the clusters IB.

\section{Percolation Model of Composite Structure}

The increase of concentrations of material subsystems certain elements in the stochastic process of its genes is and evolution leads to initiation of a connected region of a certain phase (PC), which penetrates the physical body. This causes a qualitative leap in the development of the structure - the implementation of the structural phase transitions $[3,4,11,12]$. One of the characteristic dimensions of the percolation cluster at the transition becomes comparable to the dimensions of the physical body. This 
leads to an abrupt change of the correlation length in the material, the appearance of a preferred direction and the lowering of the symmetry of the object. Thus, the percolation cluster, which sometimes takes a fraction of one percent of the body[13], do not just change some of its physical and chemical parameters, but in essence, defines the new state of matter[14].

The model is solved continuum percolation problem. Simulation of cluster formation processes is carried out on a square field of size $370 \times 370$ standard units of length, the elements of a percolation cluster - circle whose diameter is in the process of model experiment is fixed, or chosen from a set of values having a normal distribution. Control parameters of the model are the ratio between the diameter of the particles and the side of the field, as well as between the sizes of the particles forming the clusters. In model experiments, generally, the side of the square of the field is 40-50 times greater than the diameters of the particles. The coordination spheres consistent increase has been selected as the algorithm of cluster growth. Random Number Generation (RNG) with a uniform distribution[15] plays an important role in this process. The coordinates of cluster centers are selected using RNG, then - one of the centers, which will be another act of filling, and, finally, the generation indicates a place in the filled area, which be located next part of the growing cluster. RNG determines the specific value of the particle size, which are in the given interval.

The model clusters in the process of growth reach the size where ord inary particles can no longer be attached. The distance between the clusters at the same time becomes comparable to the particle diameter. This feature, as well as the fact that the geometry of the structures do not allow a new particle to lie on the plane, gives rise to the surface of the gaps and ways of arbitrary shape and size (Figure 1). This approach is actually formed by two cluster systems the particle and the internal boundaries, which are the background for each other, as in mosaic by M. Escher. (In contrast to the "white" and "black" clusters, discussed in [11], IB forms the PC, which belongs to the known class of percolation theory problems with a zero threshold[13]).

The result of model calculations for PC and largest of small cluster (LSC) are presented in Tables 1 and 2. Tables demonstrate of the model capabilities: presented the calculated parameter and theirs accuracy. These findings the result of statistical processing of the two types of computer experiments. In the first experiment, according to the previously described algorithm, increase the cluster of circles of fixed diameter around the centre of a random clustering was carried out. In the second experiment, the diameter was chosen with the help RNG with uniform distribution. Statistical sampling was done from Gaussian set of diameters, the largest of which is equal to the diameter from the experiment with the same circles. The dashes in the right column in Table 1 are typical: the onsets of clusters are extremely rare in such a setting of plane percolation problem, $\mathrm{h}$ is absence is typically.
Two-dimensional problem is easily generalized to three dimensions. The volume problem is implemented in a cube containing $10^{6}$ cells. The typical implementation of the cluster structures in three-dimensional model: percolation clusters of spheres and internal boundaries (for clarity, in an empty field) are shown in Figure 2.
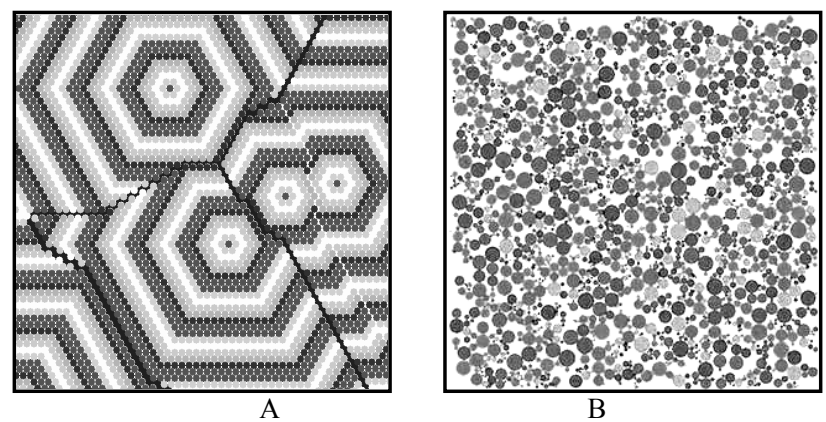

Figure 1. Cluster systems created from a set of equal particles (A) and particles with a normal size distribution (B)
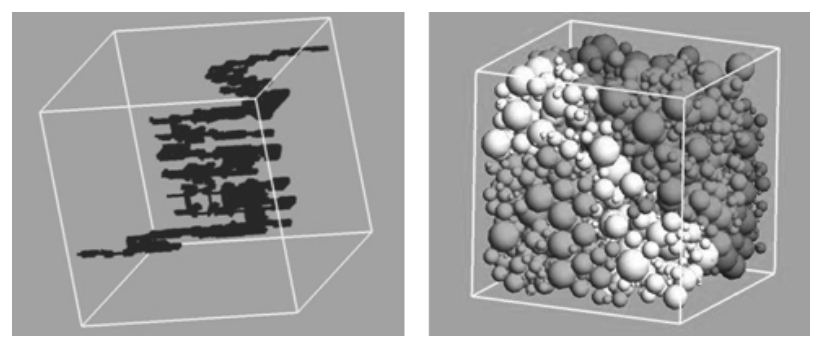

Figure 2. The cluster system of internal boundaries and spheres in three-dimensional model

Table 1. Characteristic Parameters of a Particles Clusters and Internal Boundaries in the Two-Dimensional Model

\begin{tabular}{|c|c|c|}
\hline Parameters & Fixed diameter & $\begin{array}{c}\text { Random } \\
\text { diameter }\end{array}$ \\
\hline $\begin{array}{c}\text { Power of the particles } \\
\text { clustering system }\end{array}$ & $(397 \pm 20) \cdot 10^{-3}$ & $(581 \pm 54) \cdot 10^{-3}$ \\
\hline $\begin{array}{c}\text { Fractal dimension } \\
\text { of the particles PC }\end{array}$ & $1.32 \pm 0.09$ & - \\
\hline $\begin{array}{c}\text { Radius-vector of center } \\
\text { of mass for particles } \\
\text { LSC }\end{array}$ & $286.47 \pm 26.82$ & $315.38 \pm 32.06$ \\
\hline $\begin{array}{c}\text { Radius-vector of center } \\
\text { of mass for particlesPC }\end{array}$ & $288.14 \pm 23.38$ & - \\
\hline $\begin{array}{c}\text { Value of the particle } \\
\text { SC radius gyration }\end{array}$ & $84.79 \pm 9.65$ & $25.28 \pm 2.61$ \\
\hline $\begin{array}{c}\text { Correlation length } \\
\text { of the particles clusters }\end{array}$ & $149.17 \pm 13.68$ & $44.07 \pm 4.73$ \\
\hline $\begin{array}{c}\text { Mass-to-gyration } \\
\text { radius of particles } \\
\text { PC ratio }\end{array}$ & $10.41 \pm 1.05$ & - \\
\hline $\begin{array}{c}\text { Mass-to-gyration } \\
\text { radius of particles } \\
\text { LSC ratio }\end{array}$ & $10.13 \pm 1.06$ & $9.95 \pm 1.21$ \\
\hline Power ofthe LSC IB & $(29.3 \pm 2.8) \cdot 10^{-3}$ & $(20 \pm 1.8) \cdot 10^{-3}$ \\
\hline $\begin{array}{c}\text { Power ofthePC IB } \\
\text { LSC IB }\end{array}$ & $(38.4 \pm 3) \cdot 10^{-3}$ & - \\
\hline $\begin{array}{c}\text { Gyration radius of the } \\
\text { PC IB }\end{array}$ & $93.08 \pm 9.99$ & $36.05 \pm 3.44$ \\
\hline $\begin{array}{c}\text { Gyration radius of the } \\
\text { (16) }\end{array}$ & $116.74 \pm 14.03$ & - \\
\hline
\end{tabular}


Table 2. Characteristic Parameters of a Particles Clusters and Internal Boundaries in the Three-Dimensional Model

\begin{tabular}{|c|c|c|}
\hline Parameters & $\begin{array}{c}\text { Fixed } \\
\text { diameter }\end{array}$ & $\begin{array}{l}\text { Random } \\
\text { diameter }\end{array}$ \\
\hline $\begin{array}{l}\text { Particles clustering } \\
\text { system power }\end{array}$ & $(440 \pm 50) \cdot 10^{-3}$ & $(636.9 \pm 59) \cdot 10^{-3}$ \\
\hline $\begin{array}{l}\text { Particles } \mathrm{PC} \text { fractal } \\
\text { dimension }\end{array}$ & $1.49 \pm 0.14$ & $1.39 \pm 0.12$ \\
\hline $\begin{array}{l}\text { Radius vector of the LSC } \\
\text { center of mass }\end{array}$ & $39.34 \pm 4.03$ & $36.86 \pm 3.83$ \\
\hline $\begin{array}{l}\text { Radius vector of the PC } \\
\text { center of mass }\end{array}$ & $40.73 \pm 4.48$ & $51.57 \pm 5.34$ \\
\hline $\begin{array}{c}\text { Value of the } \mathrm{SC} \text { radius } \\
\text { gyration }\end{array}$ & $20.42 \pm 2.44$ & $12.61 \pm 1.25$ \\
\hline SC correlation length & $38.58 \pm 3.72$ & $24.39 \pm 2.24$ \\
\hline $\begin{array}{l}\text { LSC degree of anisotropy } \\
\qquad A_{x y}\end{array}$ & $5.57 \pm 0.73$ & $3.89 \pm 0.41$ \\
\hline $\begin{array}{l}\text { LSC degree of anisotropy } \\
\qquad A_{x z}\end{array}$ & $5.42 \pm 0.69$ & $4.31 \pm 0.19$ \\
\hline $\begin{array}{l}\text { LSC degree of anisotropy } \\
\qquad \mathrm{A}_{\mathrm{yz}}\end{array}$ & $26.41 \pm 1.80$ & $1.93 \pm 0.10$ \\
\hline $\begin{array}{l}\text { PC degree of anisotropy } \\
\qquad A_{x y}\end{array}$ & $10.64 \pm 1.43$ & $10.02 \pm 1.35$ \\
\hline $\begin{array}{c}\text { PC degree of anisotropy } \\
A_{x z}\end{array}$ & $20.73 \pm 1.71$ & $26.39 \pm 3.25$ \\
\hline $\begin{array}{c}\text { PC degree of anisotropy } \\
A_{y z}\end{array}$ & $4.89 \pm 0.65$ & $4.86 \pm 0.62$ \\
\hline LSC IB power & $(900 \pm 100) \cdot 10^{6}$ & $(400 \pm 40) \cdot 10^{-6}$ \\
\hline PC IB power & $\begin{array}{l}(2600 \pm \\
200) \cdot 10^{6}\end{array}$ & $(800 \pm 60) \cdot 10^{-6}$ \\
\hline LSC IB gyration radius & $19.43 \pm 1.99$ & $21.28 \pm 2.37$ \\
\hline PC IB gyration radius & $29.49 \pm 3.45$ & $29.63 \pm 1.66$ \\
\hline
\end{tabular}

\section{Model of the Force Field of the Modified Sierpinski Square}

Conditions of homogeneity violation arise spontaneously in various parts of the body. Qualitatively new evolution stage of the material occurs when degree of heterogeneity increase, and there are enough collective effects. The coherent interaction between the elements of the proportionate structure (a one scale level heterogeneity) leads to configurations that act as the initial elements for higher-level structure. This provokes the inflation of structure, and thus promotes the growth the texture material inhomogeneity, leads to the hierarchical subordination, and as a consequence, to the generation of fundamentally different structures. Material and internal borders are interdependent and jointly developing cluster systems. Internal borders evolve due to redistribution of deformation in material, changing the characteristic dimensions and learning new scales, thus, modifying the material.

It is known that deformation fields essentially depend on the configuration of the inhomogeneities. In the case of quasi-linear internal borders, (we are interested in primarily), the values of the strain tensor components are proportional to $\mathrm{r}^{-1}[16]$, and, hence, their effect can be substantial at relatively large distances well above the interatomic. Orientation of the linear inhomogeneities occurring at the early stage of the material formation, of course, are not accidental, but ere not correlated.

Preferential direction in the orientation of newly formed linear irregularities occurs with increasing of their density. The deformation field activates the generation of parallel and hinders their growth in the perpendicular direction to the least extent. Thus, the linear growth of inhomogeneities occurs predominantly in two perpendicular directions, and there occurs a characteristic pattern in the distribution of equi-scale cracks. Th is aggravates the anisotropy, leads to a self-affine multifractal pattern of cracks and internal borders. A simple analogy to the plane and in the volume can be modified using affine maps such as other fractal Sierpinski carpet (square), Menger sponge, and their complements, respectively, as well as other suitable form of fractals $[4,17]$.

Consider the modified Sierpinski carpet, modeling the structure of the IB, with preferential direction (Fig. 3).

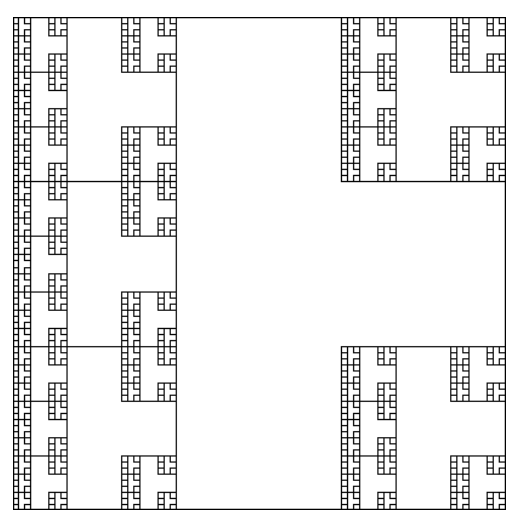

Figure 3. Modified Sierpinski carpet with mirror symmetry

We consider a "wire" model of the Sierpinski carpet. Let the initial square frame be divided by four wires into nine equal squares. The procedure is repeated many times on each of the $5^{\mathrm{m}}$ frames that are constructed in the subsequent step (see Fig.3). We also assume that on each side of frames of an arbitrary "generation", there are point sources distributed with a line density $\lambda$ and generating a field with 
the strength $E \sim 1 / r^{2}$. We find the analytic form of the force field generated by the multi-scale network of the internal edges of the Sierpinski square of an arbitrary subdivision step $\mathrm{m}$.

We assume that the origin does not lie on any line containing edges of cells of the carpet of the $\mathrm{m}^{\text {th }}$ generation. Let the carpet center be at the point $(\xi ; \eta)$.

$$
\begin{aligned}
& \text { We set } \\
& E_{\mathrm{x}} \equiv X_{m}(\xi ; \eta), E_{\mathrm{y}} \equiv Y_{m}(\xi ; \eta), \\
& \xi(n, p)=\xi+(-1)^{n} p, \quad \eta(n, p)=\eta+(-1)^{n} p, \\
& h=H / 3^{m}, m \in \mathrm{N}, \\
& A(u ; v)=k \lambda /\left(u^{2}+v^{2}\right)^{1 / 2}, B(u ; v)=k \lambda v / u\left(u^{2}+v^{2}\right)^{1 / 2},
\end{aligned}
$$

where $k$ is a coefficient depending on the selected set of units.

Then, the strength created by the carpet in the point with coordinates $(\mathrm{x} ; \mathrm{y})$ is determined by the following recurrent relations

$$
\begin{aligned}
& X_{n}(\xi ; n)= X_{n-1}\left(\xi-2 h_{n-1} ; \eta\right)+\sum_{i=1}^{2}\left\{(-1)^{i+1} B\left(\xi+h_{n} ; \eta\left(i, h_{n-1}\right)\right)\right. \\
&+ \sum_{j=1}^{2}\left[X_{n-1}\left(\xi\left(i, 2 h_{n-1}\right) ; \eta\left(j, 2 h_{n-1}\right)\right)+(-1)^{i}\left\{A \left(\xi\left(i, h_{n-1}\right) ;\right.\right.\right. \\
&\left.\left.\left.\left.\eta\left(j, h_{n}\right)\right)-A\left(\xi\left(i, h_{n-1}\right)-2 h_{n-1} ; \eta\left(j, h_{n-1}\right)\right)\right\}\right]\right\} ; \\
& Y_{n}(\xi ; n)= Y_{n-1}\left(\xi-2 h_{n-1} ; \eta\right)+\sum_{i=1}^{2}\left\{(-1)^{i} A\left(\xi+h_{n} ; \eta\left(i, h_{n-1}\right)\right)\right. \\
&+\sum_{j=1}^{2}\left[Y_{n-1}\left(\xi\left(i, 2 h_{n-1}\right) ; \eta\left(j, 2 h_{n-1}\right)\right)+(-1)^{i}\left\{B \left(\eta\left(j, h_{n-1}\right) ;\right.\right.\right. \\
&\left.\left.\left.\left.\xi\left(i, h_{n-1}\right)-2 h_{n-1}\right)-B\left(\eta\left(j, h_{n}\right) ; \xi\left(i, h_{n-1}\right)\right)\right\}\right]\right\} .
\end{aligned}
$$

\section{Oscillatory Interaction of Different Scale Structures}

The force field of randomly located quasi-parallel linear inhomogeneities is a complex interference pattern. One of the possible mechanisms of interaction of structural inhomogeneities and exploration of their scales are also associated with the interference. When some of the material field of equi-scaled cracks add up to form a local area with the magnitude of deformation, exceeding the average value, it creates preconditions for the formation energy of larger linear inhomogeneity.

Statistical self-similarity in the location of the borders leads to some "multiplicity" in the configuration of fields, this mechanis $m$ is projected for all large scale, creating an increasingly large cracks and interfaces, covering a considerable space. In turn, the more powerful deformation fields of large linear inhomogeneities provoke their further growth by acting on smaller scale heterogeneity.

This occurs simultaneously at all scales, reveals interdependence and mutual influence of different levels of structural inhomogeneities, the existence of positive feedback and interaction between the oscillating linear inhomogeneities of different structural levels. Let us examine these processes as rapidly damped oscillatory solid-state reaction. Let the physical body is an oscillatory system in this sense. This is an autonomous distributed non-conservative system with damped anharmonic vibrations.

If we assume that the generalized force of resistance acting in the system which is proportional to the velocity of propagation of energy in the system between linear inhomogeneities of different scale levels, adjusted for the level of validity of assumptions, the equation of motion (in a linear approximation) will have a standard form

$$
\mathrm{x}^{\prime \prime}+2 \gamma \mathrm{x}^{\prime}+\omega^{2} \mathrm{x}=0
$$

where $\gamma$ - the generalized damping coefficient, $\omega-$ cycling frequency. Let us estimate the conditional period $\mathrm{T}$ of these oscillations [18] for a system of linear irregularities cuboid. We introduce the inverse of the coefficient of rigidity of the body - compliance $\mathrm{C}=1 / \mathrm{k}$, which determines how far the scale of the process may spread to crack, and for which there is a calculation formula

$$
\mathrm{C}=8 \mathrm{a}^{3} /\left(\mathrm{Eh}^{3} \mathrm{~b}\right)
$$

where $\mathrm{a}$ - crack length, $\mathrm{h}$ - the distance from it to the edge of the body, b - thickness of the plate, E - Young's modulus[19].

Assuming $\gamma$ s mall, we have

$$
\omega^{2} \approx \omega_{0}^{2}=1 /(\mathrm{m} \cdot \mathrm{C})
$$

then

where $m-$ mass of the body.

$$
\mathrm{T}=4 \pi\left(2 \mathrm{a}^{3} \mathrm{~m} / \mathrm{Eh}^{3} \mathrm{~b}\right)^{1 / 2},
$$

Another evaluation of the conditional period may be obtained by determining the logarithmic decrement a serial (with an interval of period) the energy values of $\mathrm{W}_{\mathrm{n}}[18,20]$

$$
\mathrm{T}=(1 / \gamma) \cdot\left(W_{\mathrm{n}}-W_{\mathrm{n}+1}\right) /\left(W_{\mathrm{n}}+W_{\mathrm{n}+1}\right) .
$$

A possible application of the results obtained is the description of oscillatory interaction for multiscale internal boundaries in a heterogeneous material.

\section{Conclusions}

Understanding of the similarity of properties of particles and IB clusters, the ability to calculate their parameters and force interaction, can enhance the opportunities for influence on the design of materials.

\section{REFERENCES}

[1] H. Haken, Synergetics, Berlin: Springer-Verlag, Germany, 1978.

[2] N. V. Zmitrenko, A. P. Michaylov, The Heat Inertia, Moscow: Znanie, Russia, 1982. (In Russian).

[3] I. M. Sokolov, "Dimensionalities and other Geometric Critical Exponents in Percolation Theory", Soviet Physics-Uspekhi, vol. 29, pp.924-945, 1986.

[4] J. Feder, Fractals, New York: Plenum Press, USA, 1988.

[5] V. I. Solomatov, V. N. Vyrovoy, A. N. Bobrishev, 
Polystructural Theory of Composite Building Materials, Tashkent: FAN, Russia, 1991. (In Russian).

[6] A. Herega, Genesis of Structure and Properties of the Building Composites, Thesis, 2011. (In Russian).

[7] A. Herega, V. Vyrovoy, Computer Simulation of the Internal Interfaces as Elements of Material Structure, in Proceedings of the Conference "Modelling 2008", pp. 195-199, 2008. (In Russian).

[8] A. C. Varzi, Boundaries, Continuity and Contact. Nous 31, 26-58, 1977.

[9] A. I. Uyomov, System and its Parameters, In "Problems of Formal Analysis of Systems", Moscow: Vishaya shkola, Russia, 1968. (In Russian).

[10] L. von Bertalanffy, 1962, "General System Theory. A Critical Review", General Systems 7, 1-20.

[11] B. I. Shklovskii, A. L. Efros, Electronic Properties of Doped Semiconductors, Heidelberg: Springer, Germany, 1984.

[12] A. A. Berlin, S. A. Wolfson, V. G. Oshmian, N. S. Yenikopolov, Principles of a Composite Polymeric Material, Moscow: Khimiya, Russia, 1990. (In Russian).

[13] S. A. Trugman, A. Weinrib, "Percolation with a Threshold at
Zero: a New Universality Class", Physical Review B, vol. 31, no. 5, pp.2974-2980, 1985.

[14] E. F. Mikhailov, S. S. Vlasenko, "The Generation of Fractal Structures in Gaseous Phase", Physics-Uspekhi, vol. 38, pp.253-271, 1995.

[15] I. M. Sobol, Monte Carlo Method, Moscow: Nauka, Russia, 1968. (In Russian).

[16] L. D. Landau, E. M. Lifshitz, Theory of elasticity, Course of Theoretical Physics, vol. 7, Pergamon Press, 1970.

[17] A. N. Herega, N. G. Drik, A. P. Ugol'nikov "Hybrid Ramified Sierpinski Carpet: Percolation Transition, Critical Exponents, and Force Field", Physics-Uspekhi, vol. 55, pp.519-521, 2012.

[18] L. I. Mandelshtam, Lectures on some problems of the theory of oscillations (1944), in Complete Collection of Works, vol. 5, Moscow: Academy of Sciences, 1950, pp. 428-467 (in Russian).

[19] D. Broek, Elementary engineering fracture mechanics, Kluver Academic Publishers Group, Dordrecht, Boston, Lancaster, 1986

[20] A. A. Andronov, A. A. Witt, S. E. Chajkin, Theory of oscillator, L., 1966. 